\title{
Positive Regulation of Necrotic Process
}

National Cancer Institute

\section{Source}

National Cancer Institute. Positive Regulation of Necrotic Process. NCI Thesaurus. Code C40726.

Any process that increases the frequency, rate or extent of necrosis. 